\title{
Lab-made accessible full-field optical coherence tomography imaging system
}

\author{
Sistema accesible de tomografía óptica de coherencia de campo \\ completo para el laboratorio
}

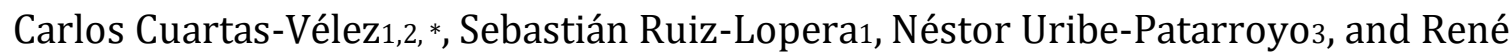 \\ Restrepo1,4 \\ 1. Applied Optics Group, Universidad EAFIT, Carrera 49\# 7 Sur-50, Medellín, Colombia. \\ 2. Biomedical Photonic Imaging Group, Technical Medical Centre, University of Twente, P.O. Box 217, \\ 7500AE Enschede, The Netherlands. \\ 3. Wellman Center for Photomedicine, Harvard Medical School and Massachusetts General Hospital, 40 \\ Blossom Street, Boston, Massachussets 02114, USA. \\ 4. Aerospace Optics Instrumentation Division, National Institute of Aerospace Technology - INTA, Ctra \\ de Ajalvir, Km 4, Torrejón de Ardóz, 28850 Madrid, Spain \\ ${ }_{(*)}$ E-mail: ccuarta1@eafit.edu.co
}

Received / Recibido: 11/04/2018 Accepted / Aceptado: 24/09/2019

DOI: $10.7149 /$ OPA.52.3.50308

\begin{abstract}
:
In this work, an easy to understand optical system was developed for the study of the medical imaging technique optical coherence tomography (OCT). This technique allows volumetric reconstruction of inhomogeneous, non-transparent and scattering samples such as biological tissues. The implementation was based on using non-specialized components for OCT under the scheme of a Michelson interferometer with a CCD camera to capture the interference patterns in a configuration known as full field OCT (FFOCT). Our system, explained in detail, was designed using components commonly found in most optical labs because our focus is to provide an accessible experimental setup for understanding the basics of OCT. The developed system possesses an axial resolution of $1.74 \mu \mathrm{m}$ and a lateral resolution of $4.5 \mu \mathrm{m}$. With the described system, tomograms of two samples were obtained: a metal coin and an ex-vivo insect wing of the blattodea family.
\end{abstract}

Key words: optical coherence tomography, full-field optical coherence tomography, interferometry, tissue, medical imaging.

\section{RESUMEN:}

En este trabajo se desarrolló un sistema óptico fácil de entender para el estudio de la técnica de imagen médica conocida como tomografía óptica de coherencia (OCT). Esta técnica permite la reconstrucción volumétrica de muestras inhomogéneas, opacas y esparsivas tales como los tejidos biológicos. La implementación se realizó usando componentes no diseñados específicamente para OCT, bajo el esquema de un interferómetro de Michelson utilizando una cámara CCD para la captura de los patrones de interferencia en una configuración conocida como OCT de campo completo (FFOCT). El sistema, explicado en detalle, se diseñó usando componentes ópticos comúnmente encontrados en los laboratorios de óptica, ya que nuestro enfoque es brindar una aproximación accesible para entender las bases de OCT. El sistema obtenido posee una resolución axial de $1.74 \mu \mathrm{m}$ y resolución lateral de 4.5 $\mu \mathrm{m}$. Con el sistema descrito se obtuvieron tomogramas de dos muestras: una moneda metálica y un ala de insecto ex-vivo de la familia blattodea.

Palabras clave: tomografía óptica de coherencia, tomografía óptica de coherencia de campo completo, interferometría, tejido, imagen médica. 


\section{REFERENCES AND LINKS / REFERENCIAS Y ENLACES}

[1] D. Huang, E. A. Swanson, C. P. Lin, J. S. Schuman, W. G. Stinson, W. Chang, M. R. Hee, T. Flotte, K. Gregory, C. Puliafito, and J. G. Fujimoto. "Optical coherence tomography”, Science 254(5035), 1178-1181 (1991).

[2] J. G. Fujimoto, C. Pitris, S. A. Boppart, and M. E. Brezinski. "Optical coherence tomography: an emerging technology for biomedical imaging and optical biopsy”, Neoplasia 2(1-2), 9-25 (2000).

[3] P. H. Tomlins, and R. K. Wang. "Theory, developments and applications of optical coherence tomography”, J Phys D Appl Phys 38(15), 2519-2535 (2005).

[4] I. Bussel, G. Wollstein, and J. S. Schuman. “OCT for glaucoma diagnosis, screening and detection of glaucoma progression”, Brit J Ophthalmol 98(Suppl 2), ii15-ii19 (2014).

[5] W. Drexler, and J. Fujimoto. Optical coherence tomography: technology and applications. New York, Springer Reference (2016).

[6] A. Dubois, and A. C. Boccara. Full-Field Optical Coherence Tomography. In: W. Drexler, J. G. Fujimoto (eds) Optical Coherence Tomography. Berlin, Heidelberg, Springer, pp. 565-591 (2008).

[7] A. Dubois, L. Vabre, A. C. Boccara, and E. Beaurepaire. "High-resolution full-field optical coherence tomography with a Linnik microscope", Appl Opt 41(4), 805-812 (2002).

[8] G. S. Kino, and S. C. Chim. "Mirau correlation microscope", Appl. Opt. 29(26), 3775-3783 (1990).

[9] L. Vabre, A. Dubois, and A. C. Boccara. "Thermal-light full-field optical coherence tomography", Appl Opt 27(7), 530-532 (2002).

[10] A. Dubois, K. Grieve, G. Moneron, R. Lecaque, L. Vabre, and C. Boccara. "Ultrahigh-resolution full-field optical coherence tomography”, Appl Opt 43(14), 2874-2883 (2004).

[11] E. Dalimier, and D. Salomon. "Full-field optical coherence tomography: a new technology for 3D highresolution skin imaging”, Dermatology 224(1), 84-92 (2012).

[12] M. Jain, N. Narula, B. Salamoon, M. M. Shevchuk, A. Aggarwal, N. Altorki, B. Stiles, C. Boccara, and S. Mukherjee. "Full-field optical coherence tomography for the analysis of fresh unstained human lobectomy specimens", J Pathol Inform 4, 26 (2013).

[13] F. Beuvon, E. Dalimier, F. Cornud, and D. N. Barry. "Full field optical coherence tomography of prostate biopsies: a step towards pre-histological diagnosis?", Prog Urol 24(1), 22-30 (2014).

[14] K. Grieve, L. Palazzo, E. Dalimier, P. Vielh, and M. Fabre. "A feasibility study of full-field optical coherence tomography for rapid evaluation of EUS-guided microbiopsy specimens", Gastrointest Endosc 81(2), 342-350 (2015).

[15] K. Grieve, M. Paques, A. Dubois, J. Sahel, C. Baccara, and J. F. Le Gargasson. “Ocular tissue imaging using ultrahigh-resolution, full-field optical coherence tomography", Invest Ophth Vis Sci 45(11), 4126-4131 (2004).

[16] M. Akiba, N. Maeda, K. Yumikake, T. Soma, K. Nishida, Y. Tano, and K. P. Chan. "Ultrahigh-resolution imaging of human donor cornea using full-field optical coherence tomography”, J Biomed Opt 12(4), 041202 (2007).

[17] O. Assayag, K. Grieve, B. Devaux, F. Harms, J. Pallud, F. Chretien, C. Boccara, and P. Varlet. "Imaging of non-tumorous and tumorous human brain tissue with full-field optical coherence tomography", Neuroimage-Clin 2, 549-557 (2013).

[18] A. F. Fercher. "Optical coherence tomography”, J Biomed Opt 1(2), 157-174 (1996).

[19] D. Gaskill. Linear systems, Fourier transforms, and optics. Toronto, John Wiley \& Sons (1978).

[20] S. S. Chim, and G. S. Kino. "Three-dimensional image realization in interference microscopy", Appl Opt 31(14), 2550-2553 (1992).

[21] L. Deck, and P. De Groot. "High-speed noncontact profiler based on scanning white-light interferometry", Appl Opt 33(31), $7334-7338$ (1994).

[22] K. G. Larkin. "Efficient nonlinear algorithm for envelope detection in white light interferometry", J Opt Soc Am 13(4), 832-843 (1996).

[23] H. M. Subhash. "Full-field and single-shot full-field optical coherence tomography: A novel technique for biomedical imaging applications", Adv Opt Technol 2012, 26 (2012).

[24] M. Akiba, K. P. Chan, and N. Tanno. "Full-field optical coherence tomography by two-dimensional heterodyne detection with a pair of CCD cameras", Opt Lett 28(10), 816-818 (2003). 
[25] D. Malarca. Optical Shop Testing. Hoboken, New Jersey, John Wiley \& Sons (2007).

[26] E. Bordenave, E. Abraham, G. Jonusauskas, N. Tsurumachi, J. Oberlé, C. Rullière, P. E. Minot, M. Lassègues, and J. E. S. Bazeille. "Wide-field optical coherence tomography: imaging of biological tissues", Appl Opt 41(10), 2059-2064 (2002).

[27] Z. Wang, and B. Han. "Advanced iterative algorithm for phase extraction of randomly phase-shifted interferograms", Opt Lett 29, 1671-1673 (2004).

[28] P. Xiao, V. Mazlin, K. Grieve, J.-A. Sahel, M. Fink, and A. Claude Boccara, "In vivo high-resolution human retinal imaging with wavefront-correctionless full-field OCT," Optica 5, 409-412 (2018).

[29] Geocities, "Insect wings", visited on September 10, 2019. www.geocities.ws/brisbane_insects/InsectWings.html.

\section{Introduction}

Optical coherence tomography (OCT) is a high-resolution (in the order of $1-15 \mu \mathrm{m}$ ) medical imaging technique used in biophotonics and medicine to produce cross-sectional and volumetric images of biological tissues. Time-domain OCT measures backscattered light from the sample by using low-coherence interferometry, in which interference is only visible if the optical path length difference between the reference and sample arm is smaller than the coherence length of the source [1]. OCT has the main advantage of not requiring prior excision of tissue, enabling so-called optical biopsies [2]. Among the ophthalmology community [3-4], OCT has been established as the gold-standard for the detection and tracking of retinal pathologies in the clinic.

In some of the time-domain OCT configurations backscattered light is measured under a Michelson interferometer scheme: a broadband source is split into two arms, one of which is propagated to a reference mirror and is called reference arm; and the other is directed onto the sample, where it is scattered, and is known as sample arm [5]. Because of the broadband source, an interferometric pattern is only visible when the time delay, or equivalently, the optical path difference, is within the coherence gate of the source [5]. This determines the axial resolution of the system: a broader bandwidth has a short coherence time, therefore increasing the axial resolution of the system; conversely, a small bandwidth increases the coherence time thus reducing axial resolution. Backscattered light is measured while displacing the reference mirror axially (in the same direction as the beam propagates) in order to obtain an interference pattern that varies in depth, creating an intensity-depth profile. This profile for a given lateral location (perpendicular to beam propagation) in the sample, is known as an "A-line" or "A-scan" and, in general, is acquired by a photodiode. The envelope of the interferometric signal contained in an A-line determines the sample reflectivity, which is the quantity of interest in OCT [5]. Cross-sectional images or "B-scans" are produced by capturing a sequence of A-lines while scanning the sample laterally, such as with galvanometric mirrors that displace the beam in one- or two-orthogonal directions perpendicular to the beam propagation. In contrast to OCT, full-field OCT (FFOCT) replaces the photodiode, and consequently, the lineper-line detection, by a CCD camera that allows the recording of two-dimensional interferometric patterns without the need for lateral displacements of the beam. Such images are also called en-face views (twodimensional lateral images) [6]. Therefore, a sequential acquisition of two-dimensional images substitutes the lateral displacements of the scanning mirror.

In OCT the lateral resolution and depth of field (DOF) are determined by the focusing optics, therefore a reasonable compromise between resolution and DOF is typically selected: lateral resolution is decreased to improve the DOF. This leads to lateral resolutions in the order of $15 \mu \mathrm{m}$ within a DOF of $\sim 2 \mathrm{~mm}$ [5]. Increasing lateral resolution in OCT leads to smaller DOF, meaning that the effective axial range in which the sample can be scanned (without implementing a refocusing system in the focusing optics) is also reduced. FFOCT implementations known as optical coherence microscopy (OCM) use high-numerical apertures, increasing the lateral resolution at the expense of reducing the DOF and thus the axial scanning range [7]. In general, OCM employs a Linnik interference microscope: a Michelson interferometer with ideally identical microscope objectives in both arms [8].

First measurements with FFOCT demonstrated its capability to produce cross-sectional and volumetric images of ex-vivo botanical samples with micrometric $(\sim 1 \mu \mathrm{m})$ lateral resolution $[9,10]$, while maintaining high axial resolution $(\sim 1 \mu \mathrm{m})$, with the drawback of a short DOF $(\sim 500 \mu \mathrm{m})$, by using a broadband source in which the illumination scheme is equivalent to that of a microscope: a homogeneous illumination field 
produced by a Koehler illuminator. Ex-vivo and in-vivo studies have been conducted in skin [11], pulmonary [12], urologic [13] and gastrointestinal tissues [14], retina and cornea [15, 16], and brain tissue [17].

Given the growing interest in research related to OCT, and the complexity involved in the development of a fully functional OCT imaging system, in this work we present a simple and easy to understand FFOCT system that is intended to be used in a context of higher education post-graduate programs in order to explain the foundations of OCT, while allowing the students to understand the concept and working mechanism of OCT. Herein, we detail the components, distances and processing techniques used to retrieve the sample information, while using optical components commonly found in most optical labs. The optical setup is built on a white-light Michelson interferometer, with a piezoelectric actuator in the reference arm in order to axially scan the sample for further volumetric imaging. By using a CCD camera, two-dimensional interferometric patterns are recorded, while the scan is performed with the piezoelectric actuator. Instead of imparting a continuous Doppler shift to the reference mirror, the envelope of the acquired interferograms - sample reflectivity - is obtained using an iterative phase-shifting algorithm where randomly generated phase shifts are used to correctly process the interferometric patterns. The optical system was successfully tested with samples of different optical properties: a metal coin and an ex-vivo insect wing of the blattodea family. Moreover, this system is readily extensible for future improvements and applications in FFOCT.

\section{Theory}

In time-domain OCT, low-coherence interferometry is used to axially scan the sample under a Michelson interferometer scheme as shown in Fig. (1), in which displacement of the reference mirror changes the optical path difference, therefore producing interference only within the coherence length of the source. Because interference is only visible within the coherence gate of the source, the axial resolution is determined by the coherence length $l_{c}$. Assuming that the spectrum of the source $S(k)=\frac{1}{\Delta k \sqrt{\pi}} \exp \left(\left[\frac{k-k_{0}}{\Delta k}\right]^{2}\right)$ has Gaussian shape in wavenumber $k$, with $k_{0}$ the central wavenumber and $\Delta k$ half the spectral bandwidth at $1 / e$ of its maximum, the coherence length defined by the full-width at half the maximum (FWM) is [5]

$$
l_{c}=\frac{2 \sqrt{\ln (2)}}{\Delta k}=\frac{2 \ln (2)}{\pi} \frac{\bar{\lambda}^{2}}{\Delta \lambda}
$$

where $\bar{\lambda}$ is the central wavelength and $\Delta \lambda$ is the FWHM of the spectrum. In order to model biological tissues, it is assumed that light is backscattered by different structures in the sample, those structures (cellular organelles, nuclei, extra-cellular tissue) are characterized by changes in the index of refraction, producing dispersion and scattering [5]. We first model the reflectivity profile of the sample $r_{s}$ as a function of depth $z_{s}$ by noticing that scattering appears when abrupt changes in the refractive index occurs. The presence of $\mathrm{N}$ interfaces between $N+1$ layers of different refractive indices is modeled as delta functions $\delta$ with specific reflectivity $r_{s_{n}}$ at a given depth $z_{s_{n}}$, given by

$$
r_{s}\left(z_{s}\right)=\sum_{n=1}^{N} r_{s_{n}} \delta\left(z_{s}-z_{s_{n}}\right) .
$$

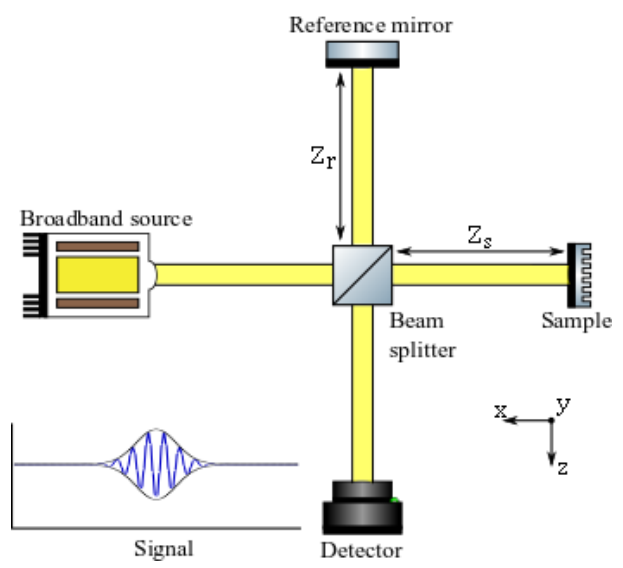

Figure 1. Michelson interferometer scheme. 
Now, we model the interferometric signal arriving at the detector by first analyzing the beam emitted by the source. Let $E_{i}$ be the electric field from the source with amplitude $s(k, \omega)$ expressed as a function of the wavenumber $k$ and the angular frequency $\omega$ :

$$
E_{i}(k, \omega)=s(k, \omega) e^{i(k z-\omega t)} .
$$

This incident electric field is split into two arms each with half the power of the source (for simplicity) by using a beam splitter. The reference beam $E_{R}$ propagates to the reference mirror in which it gets reflected back to the beam splitter, in this case, the mirror has a reflectivity $R_{R}=\left|r_{R}\right|^{2}$. While the sample arm is scattered by the sample at the $n$-th interface with reflectance $R_{S_{n}}=\left|r_{S_{n}}\right|^{2}$. After reflection, both beams are combined in the beam splitter having electric fields $E_{R}=\frac{E_{i}}{\sqrt{2}} r_{R} e^{i 2 k z_{R}}$ and $E_{S}=\frac{E_{i}}{\sqrt{2}} \sum_{n=1}^{N} r_{s_{n}} e^{i 2 k z_{s_{n}}}$ for reference and sample arm, respectively. Finally, the interferometric pattern is described by [5]

$$
I(k, \omega)=\left|E_{R}+E_{s}\right|^{2}=\left(E_{R}+E_{s}\right)\left(E_{R}+E_{S}\right)^{*},
$$

where ${ }^{*}$ represents the complex conjugate operation. The interferometric pattern in Eq. (4) produces a current in the detector $I_{D}$ modulated by the sensor response factor $\rho$ and performs an integration over time $I_{D}(k, \omega)=\rho\langle I(k, \omega)\rangle$ as the frequency of the wave is much higher than the sampling interval of the detector. Expanding Eq. (4) yields and considering that the position of the reference mirror is also a variable $[5,18]$

$$
\begin{aligned}
I_{D}\left(k, z_{R}\right) & =\frac{\rho}{4}\left[S(k)\left[R_{R}+\sum_{n=1}^{N} R_{s_{n}}\right]\right]+\frac{\rho}{2}\left[S(k) \sum_{n=1}^{N} \sqrt{R_{R} R_{S_{n}}} \cos \left[2 k\left(z_{R}-z_{s_{n}}\right)\right]\right] \\
& +\frac{\rho}{4}\left[S(k) \sum_{n \neq m=1}^{N} \sqrt{R_{S_{n}} R_{S_{m}}} \cos \left[2 k\left(z_{s_{n}}-z_{s_{m}}\right)\right]\right] .
\end{aligned}
$$

The first of resulting terms in Eq. (5) is a constant background, the second term is known as crosscorrelation and it relates the intensity from the sample and reference arm, thus the fundamental information in OCT because $R_{S_{n}}$ is provided by this term. The third term in Eq. (5) represents the interference between light reflected from the sample itself at different depths without involvement of the reference beam, known as the autocorrelation term. This term is generally much lower in intensity than the cross-correlation term, but it can be seen in the presence of strong specular reflections in the sample and is generally considered artifactual. According to Eq. (5), it is possible to produce an interferometric pattern by changing either the optical path difference $z_{R}-z_{S_{n}}$ or the wavenumber $k$. (TDOCT) corresponds to the interferometric pattern produced by changing the optical path difference, while Fourier domain OCT analyzes the interferometric signal by means of the wavenumber.

\section{2.a. Time Domain OCT}

In TDOCT, A-lines are recorded scanning the interferometric pattern with the reference arm at different depths $z_{R}$. The full spectrum of the source is incident on the sample, meaning Eq. (5) must be integrated over the wavenumbers $k$ and the dependence is expressed as a function of the reference mirror path length $z_{R}$, yielding [5]

$$
I_{D}\left(z_{R}\right)=\frac{\rho}{4} \mathrm{~S}_{0}\left[R_{R}+\sum_{n=1}^{N} R_{S_{n}}\right]+\frac{\rho}{2} S_{0}\left[\sum_{n=1}^{N} \sqrt{R_{R} R_{S_{n}}} e^{-\left[z_{R}-z_{s_{n}}\right]^{2} \Delta k^{2}} \cos \left[2 k_{0}\left(z_{R}-z_{s_{n}}\right)\right]\right],
$$

where $S_{0}$ is the source power and a spectrum $S(k)$ with Gaussian shape is assumed [19]. Eq. (6) is composed of two terms: a background, which is independent of depth and corresponds to the total light reflected from the reference mirror and the sample, and a cosine function modulated by a Gaussian function and whose amplitude is determined by the reflectivity of the sample. The term of interest $R_{S_{n}}$ may be obtained as the envelope of each interferometric pattern in the positions $z_{s_{n}}$ as the envelope of the cosine function changes as a function of the sample reflectivity. Gaussian modulation is proportional to $R_{R}$ meaning that reference arm also increases the signal measured at the detector. This photocurrent measurement is known as A-line and corresponds to the depth-intensity profile. A typical TDOCT A-line is illustrated in Fig. (2), where four reflectors are responsible of the interference along the axial range. 


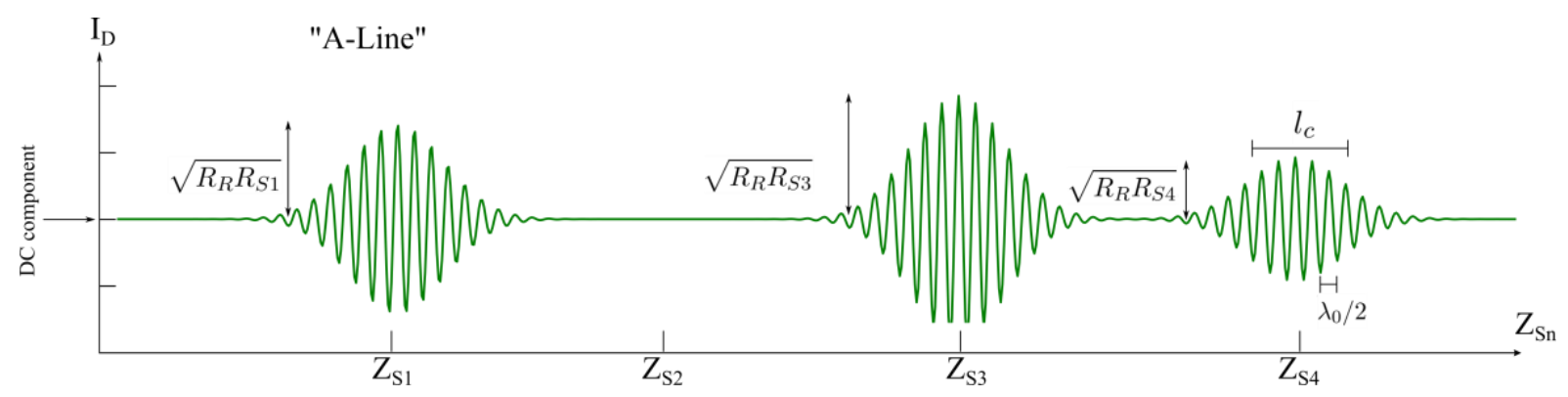

Figure 2. Time-domain OCT A-line captured by changing optical path difference.

\section{2.b. Full-Field OCT}

FFOCT uses the same principles of OCT, but it captures the transverse plane in a direct way: twodimensional interferometric patterns are recorded by a 2D sensor, hence the recorded signal at a given position $\mathrm{z}_{\mathrm{R}}$ encodes the sample reflectance $\operatorname{map} R_{s_{n}}(x, y)$ as [6]

$$
I\left(x, y \mid z_{R}\right)=\frac{I_{0}}{4}\left[R_{R}(x, y)+\sum_{n=1}^{N} R_{S_{n}}(x, y)+2 \sqrt{R_{R}(x, y) R_{S_{n}}(x, y)} \cos [\phi(x, y)+\psi]\right],
$$

where the first two terms represent the fraction of light that does not interfere, $\phi(x, y)$ is the phase difference between the reference and sample fields, and $\psi$ is a global phase shift between the reference and sample. Several en-face image reconstruction schemes have been proposed to retrieve the fringe envelope, which is proportional to the sample reflectance $R_{S_{n}}(x, y)$ [20-22]. Commonly, a discrete or continuous phase shift $\psi$ is induced, and fringe envelope is obtained by processing a set of images $I\left(x, y \mid z_{R}\right)$ [23]. In hardware approaches, two CCD cameras are combined to perform heterodyne detection [24]. In the "integrating bucket" approach, a continuous and periodic phase shift is induced while the set of images or "buckets" is recorded by integrating the intensity along fractions of the oscillation period [6]. Sinusoidal phase shift with four integrating buckets is usually used [7]. In the discrete approaches, a set of images with known phase shifts are recorded and conventional phase shifting methods [25] are used to retrieve the fringes envelope [26]. Due to inertia of the moving mirror in discrete phase shifts, continuous scheme allows higher scan speeds. Furthermore, both schemes require a stable and exact phase shift values, which demands complex instruments and control. However, the advance iterative algorithm (AIA) allows envelope extraction from randomly shifted interferograms using a minimization procedure that iteratively finds the phase, phase shifts and envelope [27]. Here, we take advantage of AIA in order to retrieve the envelope of the signal, as it does not require prior knowledge or control of the phase shifts, and therefore a flexible number of images can be used in the reconstruction. This procedure facilitates further acquisition and processing of the OCT signal, as it is easily implementable experimentally in teaching students instead of more expensive hardware solutions.

\section{2.c. Lateral resolution and depth of field}

Theoretical lateral resolution $\Delta x$ is determined by the diffraction limit of the imaging system, that depends on the numerical aperture $N A$ as in microscopy [5]:

$$
\Delta x=\frac{2 \bar{\lambda}}{\pi N A} .
$$

Fine lateral resolution can be obtained by using a large numerical aperture at the expense of reducing the depth of field, i.e. the region where images appear focused, determined by the confocal parameter $b$, which is equal to twice the Rayleigh range $z_{R}[5]$,

$$
b=2 z_{R}=\frac{\pi \Delta x^{2}}{\lambda}
$$

resulting in a trade-off between lateral resolution and depth of field; increasing one decreases the other in a quadratic way, as in Fig. (3), where the relationship between both is displayed for two different numerical apertures. The confocal effect in raster scanning systems does not exist in FFOCT, and therefore multiple scattering limits the penetration depth. However, spatially incoherent illumination has been used to reject multiply scattered light in FFOCT, effectively regaining the confocal range. Even more, it has been shown recently that with an incoherent illumination, it is possible to have a system with diffraction-limited resolution, regardless of aberrations [28]. 


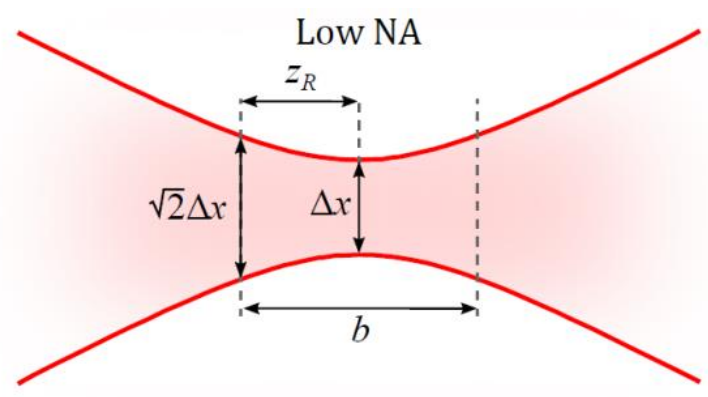

(a)

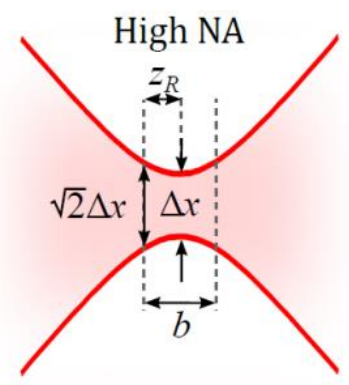

(b)

Figure 3. Lateral resolution and depth of field trade-off examples for (a) high and (b) low numerical apertures.

\section{Methods and Materials}

Based on a typical Michelson interferometer we developed the optical system shown in Fig. (4). The source is a halogen source (THORLABS OSL2) whose spectrum is presented in Fig. (5a) and has a theoretical axial resolution of $0.72 \mu \mathrm{m}$. Light emitted by the source is propagated through a collimator, where beam divergence is reduced. At the collimator output, a diaphragm is located, allowing control of beam size and granting spatial coherence to the source.
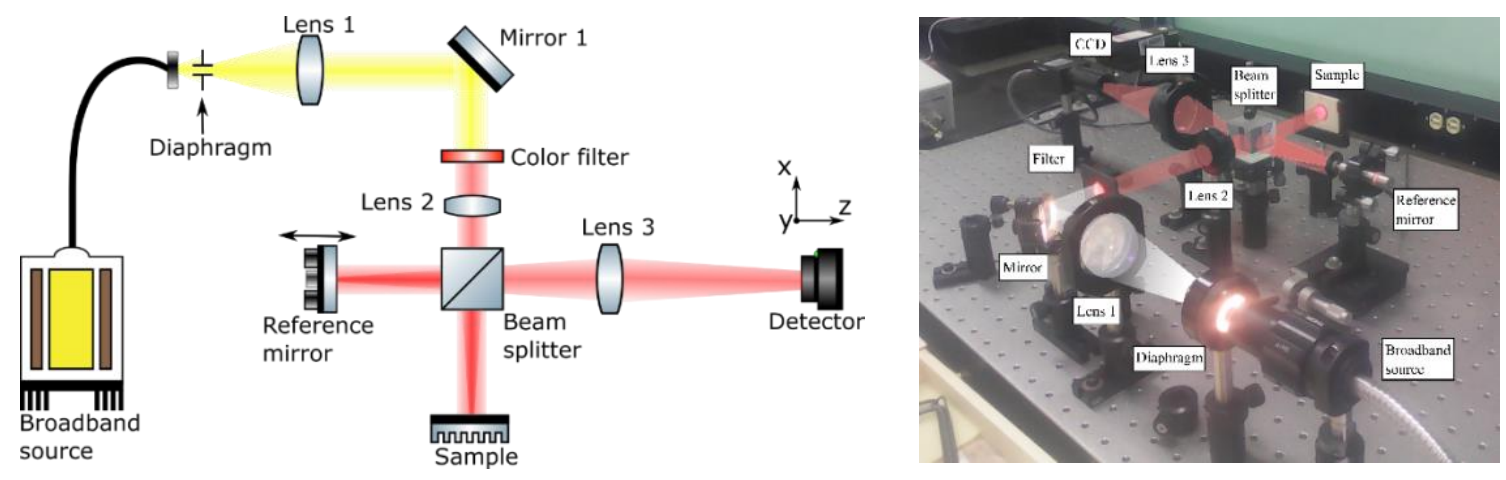

Figure 4. Schematic (left) and picture (right) of the FFOCT system.

A $\phi 2 "$ lens (THORLABS LA1050) with effective focal length $100 \mathrm{~mm}$ is placed $10 \mathrm{~cm}$ after the diaphragm, this first lens has the function of collecting light from the diaphragm. Following, a red filter (PHYWE 8406) is placed to reduce the source bandwidth by absorbing a portion of the spectrum as is shown in Fig. (5b). This filter reduces the effect of chromatic aberration produced by optical elements, relaxing the requirements for achromatic optics, however, the axial resolution is reduce to $2.14 \mu \mathrm{m}$. At $15 \mathrm{~cm}$ from the first lens a second lens $\phi 1$ " (THORLABS AC254-200-A-ML) is situated, with focal length $300 \mathrm{~mm}$. The second lens allows to focus light onto the sample plane, increasing the available light in the system. A 50/50 cube beam splitter (Edmund Optics 32-702) divides the light into the reference and sample arms. Reference beam propagates to a mirror that is coupled with a piezoelectric actuator (THORLABS MDT693A) with step $266 \mathrm{~nm} / \mathrm{V}$ and $20 \mu \mathrm{m}$ of maximum displacement. Zero-delay is positioned using a micrometric screw with $1.5 \mathrm{~cm}$ maximum travel distance and $1 \mu \mathrm{m}$ resolution. Sample and reference mirror are imaged at detector plane by $2 f$ optical setup using a $\phi 2 "$ lens (THORLABS LA1050) with effective focal length $100 \mathrm{~mm}$. Finally, a CCD camera (Point Grey Grasshopper3 GS3-U3-91S6M-C) with $3376 \times 2704$ pixels, pixel size $3.69 \mu \mathrm{m}$ and 14 bits is used to record the interferometric patterns. The CCD camera captures two-dimensional images as the reference mirror is displaced axially, thus we do not require lateral scanning of the beam. 


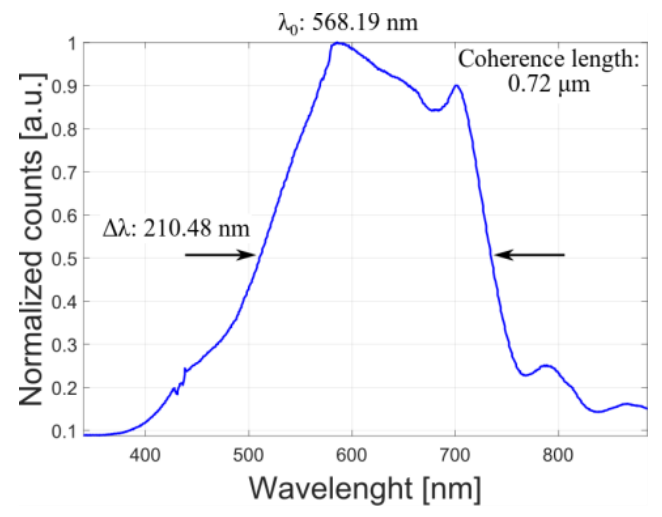

(a)

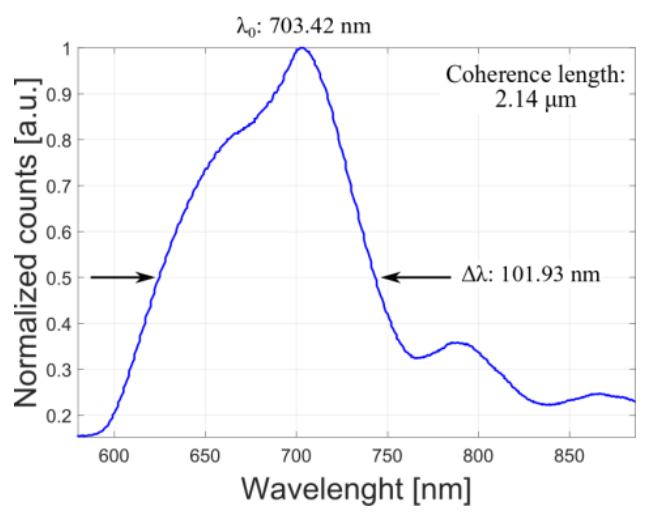

(b)

Figure 5. Source spectrum (a) without and with (b) color filter. Axial resolution was set to 2.14 $\boldsymbol{\mu m}$.

Once the two arms have an optical path difference within the coherence length of the source, interferometric patterns are obtained. Figure (6a) shows low-coherence fringes using a mirror as sample. Interferograms are processed with AIA algorithm to obtain en-face images, consisting of the fringe's envelope, using 8 images at each axial position acquired with an exposure of $\sim 15 \mathrm{~ms}$. As AIA is based on a minimization procedure, the number of images can be increased or decreased in order to improve the results, we set the tolerance of the optimization to $10^{-4}$ and 40 maximum iterations. After extraction of modulation from the interferometric pattern the corresponding modulation is presented in Fig. (6b). Axial resolution of the system can be deduced from the FWHM of an A-line signal acquired by a single pixel along all axial positions of the reference mirror. For instance, central pixel signal is shown in Fig. (6c), exhibiting an axial resolution of $1.74 \mu \mathrm{m}$, similar to the theoretical value of $2.14 \mu \mathrm{m}$ that is calculated with Eq. (1) using the spectrum properties $\bar{\lambda}=703.42 \mathrm{~nm}$ and $\Delta \lambda=101.93 \mathrm{~nm}$ shown in Fig. (5b). Additionally, theoretical lateral resolution and Rayleigh range calculated with Eq. (8) and (9) are $4.5 \mu \mathrm{m}$ and $200 \mu \mathrm{m}$, respectively, considering the experimental beam diameter of $\sim 10 \mathrm{~mm}$.

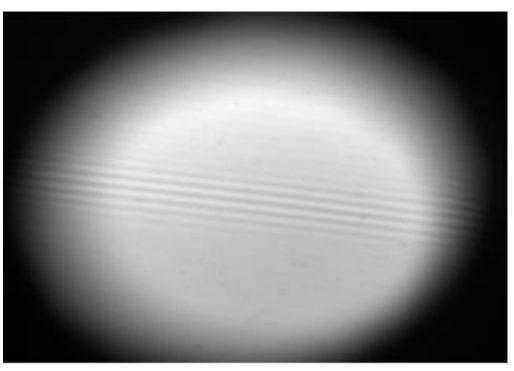

(a)

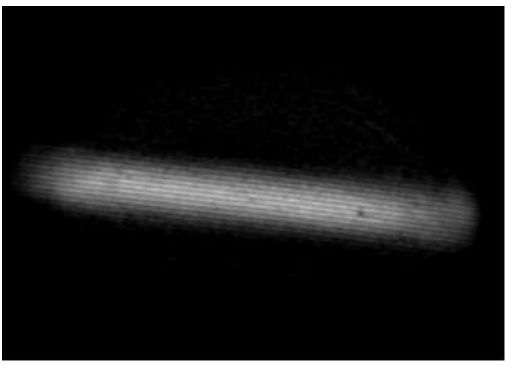

(b)

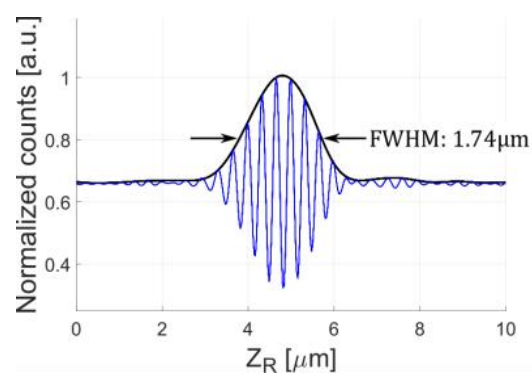

(c)

Figure 6. White-light interferometric pattern (a), corresponding modulation (b) and A-line of central pixel (c).

Detection sensitivity determines the minimum sample reflectivity that can be detected with the OCT system, corresponding to a signal-to-noise ratio equal to one; detection of weaker scattering media requires a higher sensitivity [5]. Therefore, as intensity decreases with depth, maximum axial range can be limited by sensitivity in highly scattering or absorbing media. In the ideal case, detection is shot-noise limited, meaning that electrical noise is small compared to photon noise, thus sensitivity depends mainly in well capacity of the CCD pixels [6]. We performed experimental measurements to estimate the system sensitivity by placing neutral density filters in the sample arm where a mirror is used, and we obtained a measurable interference signal up to $36 \mathrm{~dB}$. However, this could be greatly improved with the use of a different type of source, for example superliminescent diode (SLED) sources, nonetheless, an upgrade in this may incur in a significant cost. The relatively low sensitivity compared to typical values in OCT of $95 \mathrm{~dB}$ [5], is affected by thermal noise produced by electron movements due to thermal energy in the CCD materials and performance of interferograms processing algorithm. Another possible way to improve the sensitivity is by placing an attenuator in the reference beam to match lower intensities from the sample. 


\section{Experimental results}

Using the FFOCT system in a Michelson interferometer scheme described in the previous section, our idea is to expose applications of OCT with a system using non-dedicated components for OCT. We performed a volumetric reconstruction of a coin topography and a cross-sectional analysis of ex-vivo blattodea wing.

\section{4.a. Coin topography}

We first performed an experiment to evaluate proper operation of the system by evaluating the axial resolution through different depths with a highly reflective sample. Usually, a mirror is at different depths. However, to perform fist evaluations, we selected a sample with similar properties to an optical mirror but attracting more easily the attention of students. The advantage of the coin sample is that it produces a strong reflection at the surface, therefore, its topography can be used to evaluate the performance of the system while maintaining high signal through depth scan. The coin and the imaged region are shown in Fig. (7). In this kind of metallic samples high specular reflection clearly illustrates the principle behind OCT: whitelight interferometry. We jointly displace the piezoelectric actuator along the micrometric screw to scan $300 \mu \mathrm{m}$ in depth with $1 \mu \mathrm{m}$ steps, getting a raw volume of $800 \times 600 \times 300 p x^{3}$.

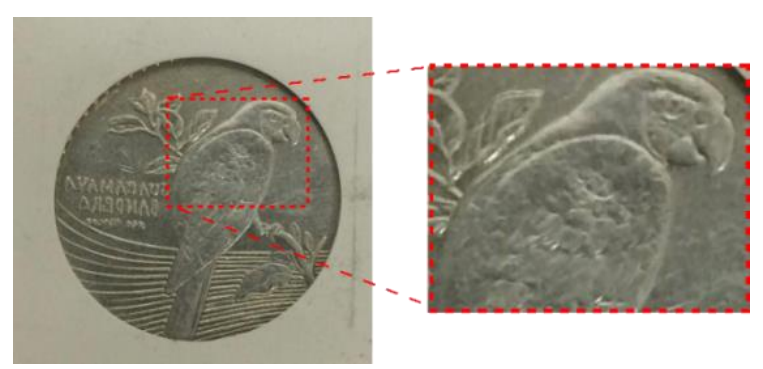

Figure 7. Coin and region of interest used in the experiment.

A sequence of captured modulations is presented in Fig. (8): it clearly shows how OCT scans regions in depth as the optical path difference is increased. The images presented in Fig. (8) were captured from left to right and from top to bottom, with a separation of $20 \mu \mathrm{m}$; they show how the interference performs a sequential scan of the different depths in the coin topography, starting with the lower area, where the characteristics of the topography is higher, appearing first in the sequence of interferograms. Further, the central area of the coin is scanned, where flat and homogeneous regions appear. The progression followed by the modulation in the images also indicates missalignments between reference and object, since the interference in flat regions of the coin is distributed along different depths [5].

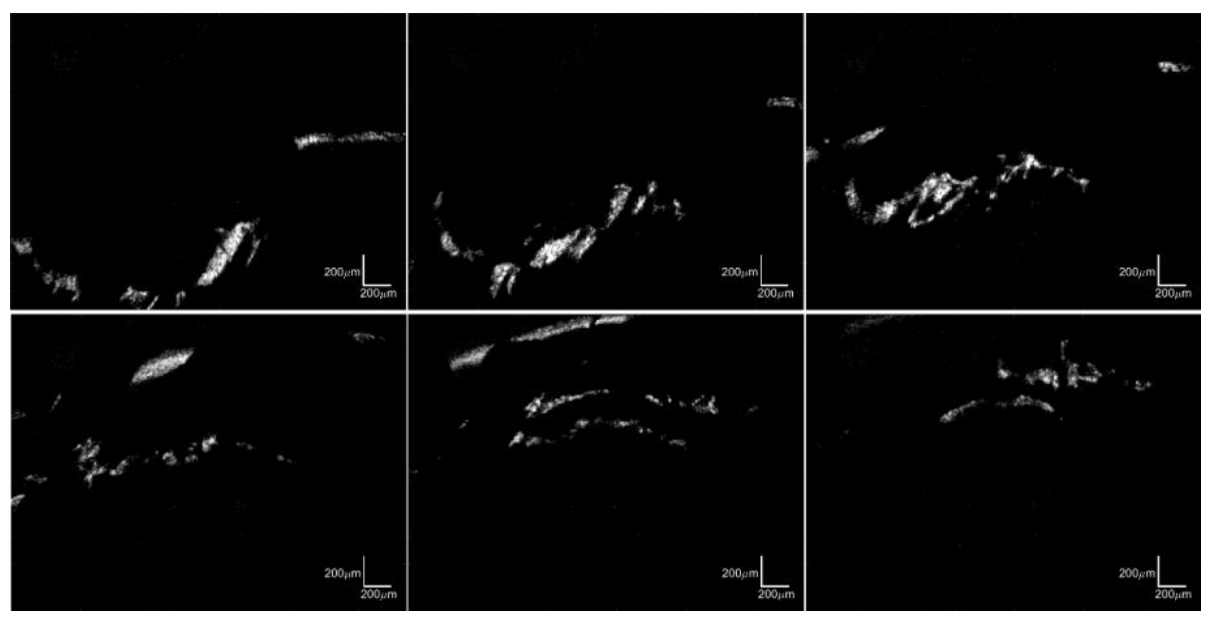

Figure 8. Sequence of modulations obtained while scanning the coin. Images where capture from left to right and up to down, with differences of $\mathbf{2 0 \mu m}$.

The final reconstruction is a sum of all depth samples and shows that total depth information is equivalent to capture a direct image of the sample presented in Fig. (9a), however, OCT allows cross-sectional views. 
Since the axial displacement and the pixel size are known, cross-sectional slices of the coin were taken showing the coin relief along the $X$ and $Y$ axis. Two cross-sectional planes $Z X$ and $Z Y$, at $Y=1.06 \mathrm{~mm}$ and $X=1.71 \mathrm{~mm}$ respectively, and indicated by white dotted lines in Fig. (9a) show some points of interest (A-H) in Fig. (9a). Figure (9b) corresponds to the cross section in the $Z X$ plane, in OCT, this plane is known as B-scan and is located at $Y=1.06 \mathrm{~mm}$. In this case, the presence of only one reflector for all depths is expected, because the coin only reflects light on the surface before absorbing or reflecting the incident light. As the scanned axial distance and the size of each step are known, we concluded that the relief of the coin has a maximum height of $50 \mu \mathrm{m}$. In the cross-section, the points (A-D) are distinguishable along the $X$ axis: point A shows a flat region along $X \sim 0-500 \mu \mathrm{m}$, followed by point B as a dark transition zone to a higher point. $\mathrm{C}$ appears as a small dark region, indicating a high scattering of light in the coin. Finally, the point $\mathrm{D}$ shows a zone with relief fluctuations for $X \sim 1350-1450 \mu \mathrm{m}$.

The cross-sectional slice in Fig. (9c) has a lower depth variation, this is related with inclination of object beam with respect to reference beam. The points $\mathrm{E}$ and $\mathrm{H}$ show two zones where the depth is constant along $Y \sim 50-150 \mu \mathrm{m}$ and $Y \sim 2000-2800 \mu \mathrm{m}$, representing flat regions. Around point $\mathrm{F}$ a small homogeneous but discontinuous area appears, due to the transition between heights in the relief. The point $G$ is the transition from the reliefed area to a flat area, appearing as a opaque point. A greater intensity in the image, represents that light has been reflected by the coin in the direction of the detector, while a low intensity, shows absortion or reflection in random directions.

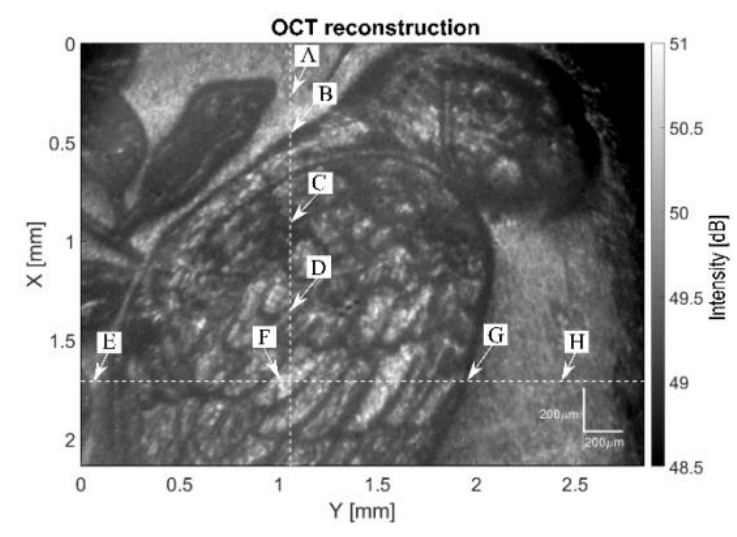

(a)

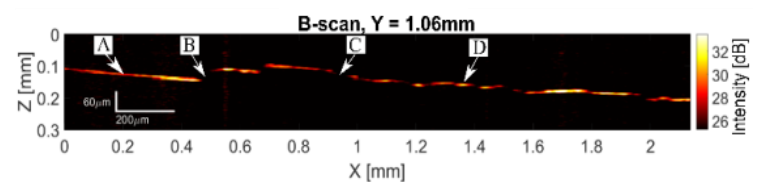

(b)

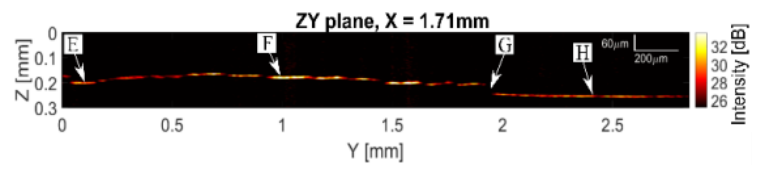

(c)

Figure 9. OCT reconstruction of the coin. a) Projection of all depths, cross-sectional b) ZY and c) ZX views of the coin.

\section{4.b. Blattodea wing}

The second sample analyzed was an ex-vivo blattodea wing. Figure (10a) shows blattodea frontal wing and imaged region. In this case, the scanning depth was set to $500 \mu \mathrm{m}$, capturing interfometric patterns spaced $1 \mu \mathrm{m}$ and using ten images for AIA with the same previous parameters. The projection of all modulations along depth is presented in Fig. (10b), some wing structures are distinguishable and indicated with arrows A-I in the cross-sectional views. Point A corresponds to a region in the sample holder, B and I corresponds to places with adhesive protector, $\mathrm{C}$ is a vessel in $X \sim 500 \mu \mathrm{m}$, appearing as a dark region since absorption in this kind of structure is expected to be high [29]. D is a region of the wing membrane observed as a bright area, where the light is highly reflected. E appears as a dark region because of high absorption, F shows a point located on the wing radius, and $\mathrm{H}$ corresponds to an interface between wing and air. Two crosssectional slices at $X=0.6 \mathrm{~mm}$ and $Y=1.72 \mathrm{~mm}$ are shown in Figs. (10c) and (10d) respectively. Figure (10c) is a B-scan from the wing. Points A-E described above are located in the figure. Points PA1 and PA2 refer to first and second surface of the adhesive protector, in general they are highly bright since this material reflects a large portion of incident light, and it is the first surface. The region RA corresponds to the location of the radius of the wing, it is expected to be a dark region because as it was shown by the direct image, it strongly absorbs light. The dark points located along the $X$ axis and denoted as VA are wing vessels, these points reflect less light than the membrane given their structural characteristics. Wing membrane is a thin structure which reflects high percentage of light and is indicated as ME. Continuous line marked as $\mathrm{PM}$ is the sample holder. 

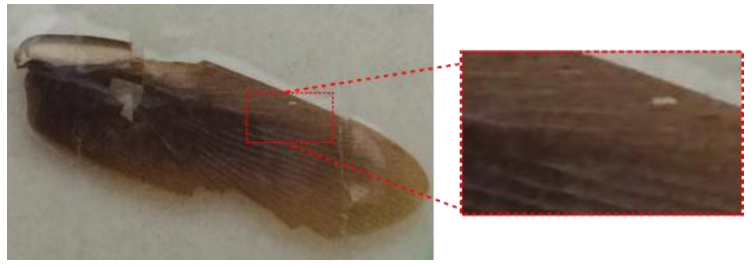

(a)

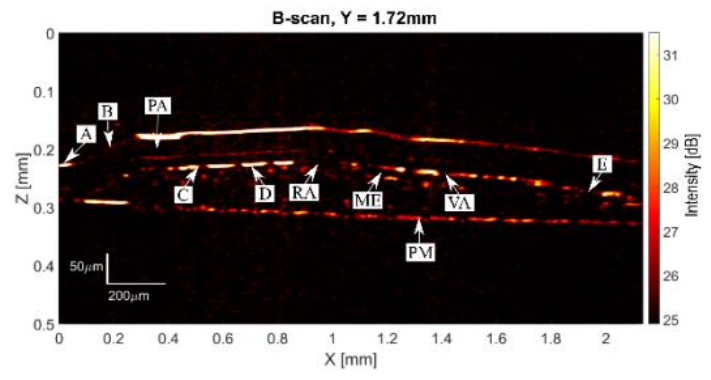

(c)

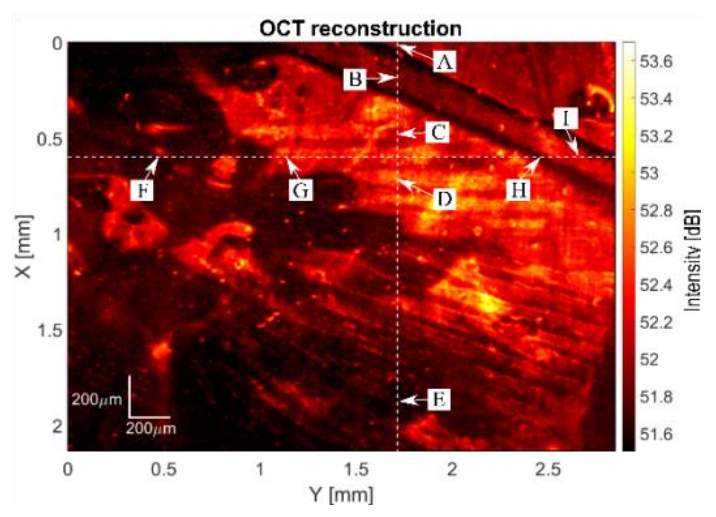

(b)

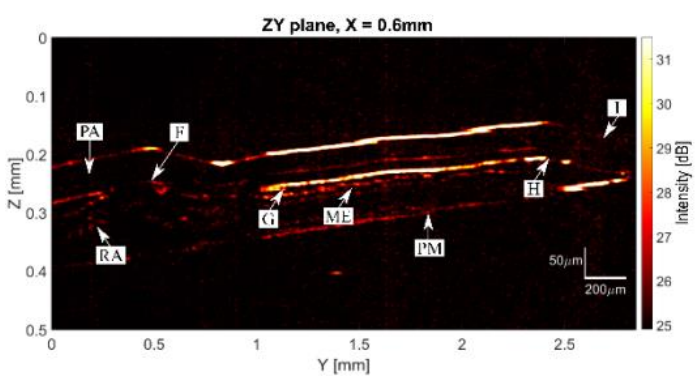

(d)

Figure 10. (a) Blattodea wing and region of interest imaged with FFOCT. OCT reconstruction: b) projection with all depth samples and cross-sectional c) ZX and d) ZY views.

Point A shows a flat region on the direct image; however, cross-sectional views allow to determine the presence of three layers, two of them corresponding to the adhesive protector and the last the sample holder. Point B, from the same previous layers, has a lower intensity since it is tilted. C shows one ramification of the radius. $\mathrm{D}$ is located on a section of the membrane. $\mathrm{E}$ is a dark region in the in the center of the wing. Fig. (10d) is the cross-section of the $Z Y$ plane at $X=0.6 \mathrm{~mm}$. The scan in this case is aligned with a region of the wing membrane F-I points located along the $X$ axis those points are comparable in with previous cross-sectional view. Note that even with the higher absorption of this sample, it was still possible to obtain structural images, which in many cases cannot be easily achievable if backscattered light is lower than absorbed light.

\section{Conclusions}

In conclusion, we have presented the physical basis and experimental details for a FFOCT system. The optical system was built using non-dedicated components for OCT and is based on a white-light Michelson interferometer scheme with and halogen lamp, where the reference arm is axially displaced to scan depth layers in samples. We obtained an axial resolution of $1.74 \mu \mathrm{m}$ and lateral resolution of $4.5 \mu \mathrm{m}$. By using an iterative phase-shifting algorithm, the modulation of interferometric patterns were retrieved, and sample reflectivy characterized. The proposed optical system was used to successfully obtain cross-sectional views of two samples: coin relief and blattodea wing structure. Visualization of the wing showed different inner structures, such as the radius, membrane and vessels. 